\title{
VAGINOSIS CITOLIITICA: UN DIAGNÓSTICO DIFERENCIAL POCO FRECUENTE DE VULVOVAGINITIS MICÓTICA A REPETICIÓN
}

\author{
Paolo Ricci A. ${ }^{1}$, Luis Contreras M. ${ }^{2}$, Luis Contreras S. ${ }^{a}$ \\ ${ }^{1}$ Unidad de Ginecología, Departamento de Obstetricia y Ginecología, ${ }^{2}$ Departamento de Anatomía Patológica, Clínica \\ Las Condes.
}

a Alumno, Escuela de Medicina, Universidad Andrés Bello.

\section{RESUMEN}

Los síntomas compatibles con vaginitis y vaginosis, son la causa más frecuente de consulta al ginecólogo. La descarga vaginal abundante y el prurito, constituyen la primera sospecha de estas patologías. Sin embargo, debemos tener presente que el aumento significativo de lactobacilos, conocido como lactobacilosis, puede expresarse también con los mismos síntomas y signos de una vulvovaginitis micótica. Si esta condición se mantiene en el tiempo, puede provocar un grado de acidez anormal en la vagina, y puede llevar a una lisis de las células epiteliales que se conoce como vaginosis citolítica. Este diagnóstico, aunque es poco frecuente, debemos sospecharlo especialmente, en aquellas mujeres que relatan tener múltiples consultas por candidiasis vaginal, con escasa o nula respuesta frente a la terapia antimicótica.

\section{PALABRAS CLAVE: Vaginosis citolítica, vulvovaginitis, lactobacilos, lactobacilosis, citólisis, flora comensal, micosis vaginal}

\section{SUMMARY}

The symptoms consistent with vaginitis and vaginosis are the most common cause of the visit to the gynecologist. Heavy vaginal discharge and pruritus are the first suspicion of this pathology. However, the significant increase in lactobacilli, known as lactobacilosis can be expressed also with the same symptoms and signs of a fungal vulvovaginitis. If this condition is maintained over time, can cause abnormal acidity in the vagina, and may lead to epithelial cell lysis, which is known as cytolytic vaginosis. This diagnosis, although rare, should be suspected especially in women who reported having multiple consultations for vaginal candidiasis, with little or no response to antifungal therapy.

KEY WORDS: Cytolytic vaginosis, vulvovaginitis, lactobacilli, lactobacillosis, citolysis, commensal flora, vaginal mycosis 


\section{INTRODUCCIÓN}

La vaginosis y vaginitis son patologías extremadamente frecuentes. Los síntomas vaginales constituyen la razón más común para consultar al ginecólogo (1). La mayoría de los casos son debido a cándida Sp. (2) y se presentan con flujo aumentado y prurito vaginal.

El lactobacilo es la bacteria que encontramos en mayor cantidad en la vagina. Su contribución principal es sobre el pH vaginal, para así mantener el equilibrio y protección contra bacterias eventualmente patógenas (3-5).

La presencia de 5 bacilos por cada 10 células escamosas en el flujo de descarga vaginal, ha sido definido como un factor protector para candidiasis vaginal (6). Otro mecanismo de protección otorgado por los lactobacilos, es la inhibición de la adherencia de la cándida a las células epiteliales vaginales, a través de la producción de sustancias antimicrobianas (peróxido de hidrógeno, bactericidas y biosurfactantes), competencia por nutrientes (arginina deiminasa) y competencia por receptores (adhesión al epitelio) (7-9). Se han identificado 20 tipos diferentes de lactobacilos en la vagina humana (10).

Debido a todo lo anterior, el lactobacilo es considerado una bacteria comensal, cuya presencia es un factor protector en la vagina. Sin embargo, se han registrado y descrito casos en la literatura médica, de mujeres con un aumento excesivo de lactobacilos, lo que se conoce como lactobacilosis (11). Esta condición provoca una acidificación extrema del medio vaginal, lo cual puede llevar a la consecuente lisis de las células epiteliales. Esta entidad patológica se conoce como vaginosis citolítica (1214) y generalmente se confunde con la vulvovaginitis micótica por cándida albicans $(13,14)$.

A continuación describimos un caso clínico correspondiente a vaginosis citolítica.

\section{Caso clínico}

Mujer de 35 años, nulípara, que consulta por flujo vaginal abundante, tipo leucorrea y prurito. Relata que ha acudido a cuatro ginecólogos durante el último año, recibiendo tratamiento en todas las consultas, por una sospecha de vulvovaginitis micótica, sin respuesta frente a los medicamentos empleados. Ha utilizado fluconazol oral, clotrimazol y miconazol-tinidazol en óvulos, pomadas de clotrimazol y nistatina, en múltiples ocasiones. La descarga de flujo es abundante y aumenta aún más en forma cíclica. En uno de los controles se encontró un ectropión, que fue tratado con crioterapia, sin embargo, no hubo disminución del flujo vaginal.
Además relata que en los últimos meses presenta sensación de quemadura en la mucosa vaginal y se ha agregado dispareunia. Relata que en algunas ocasiones el flujo forma verdaderas costras de color blanco, sobre las paredes vaginales.

Al examen ginecológico destacaba un flujo abundante de color blanco y eritema sobre las paredes vaginales.

Debido al antecedente de múltiples tratamientos con antimicóticos sin respuesta, se tomó una muestra de flujo para PCR y se realizó un examen directo al fresco, tinción de gram y cultivo. Los exámenes descartaron la presencia de cándida y bacterias como gardnerella, tricomona y clamidia, entre otras. Las pruebas demostraron solamente la presencia de lactobacilos en abundancia.

Se tomaron biopsias de la mucosa vaginal, que mostraron lisis de células epiteliales, destacando la fragmentación de células y salida de núcleos. Además de una gran cantidad de lactobacilos alargados (Figura 1 a 4).

Se midió el $\mathrm{pH}$ vaginal en ambas fases del ciclo, variando entre 3 y 4, encontrándose mayoritariamente en el valor más bajo. Debido a la clínica, examen ginecológico y pruebas de laboratorio, se cumplieron los criterios para realizar el diagnóstico de vaginosis citolítica. Se inició tratamiento para controlar la población de lactobacilos, con antibióticos. Se utilizaron duchas vaginales de bicarbonato de sodio, para controlar el $\mathrm{pH}$. Las mediciones de $\mathrm{pH}$ de control, demostraron la efectividad del tratamiento, encontrándose entre 5 y 6 mayoritariamente.

\section{DISCUSIÓN}

La vaginosis citolítica (VC) es una condición patológica muy poco frecuente, y que en la mayoría de las veces queda olvidada. Se trata de mujeres

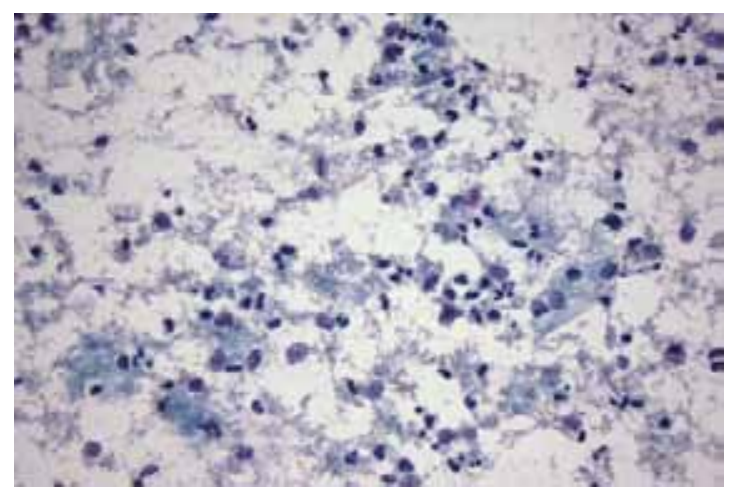

Figura 1. Frotis citológico con marcada citolisis en células escamosas y presencia de numerosos lactobacilos. Citología Papanicolaou, aumento 400x. 


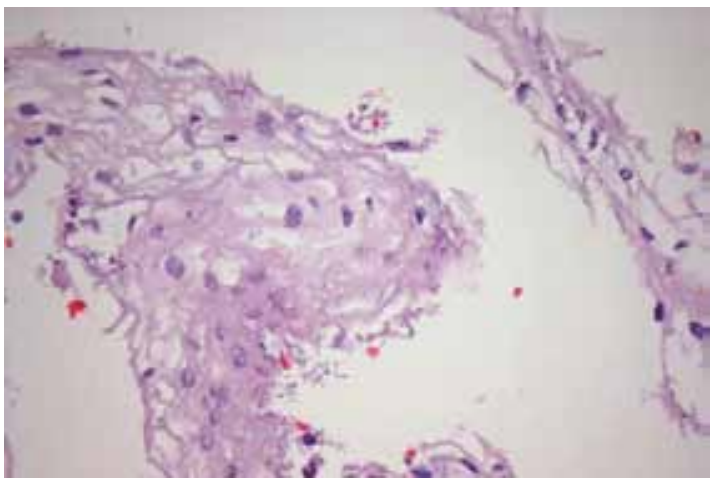

Figura 2. Corte de cuello uterino con presencia de numerosos lactobacilos sobre la superficie epitelial. Tinción Hematoxilina-Eosina, aumento 400x.

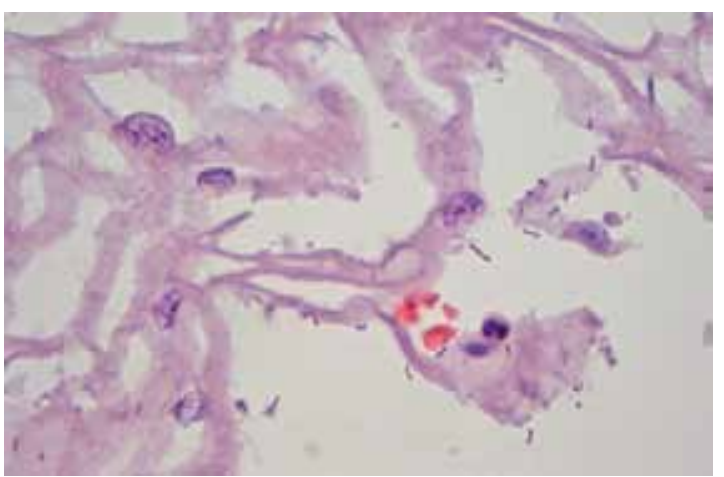

Figura 3. Corte de cuello uterino con presencia de numerosos lactobacilos sobre la superficie epitelial. Tinción Hematoxilina-Eosina, aumento 1000x.

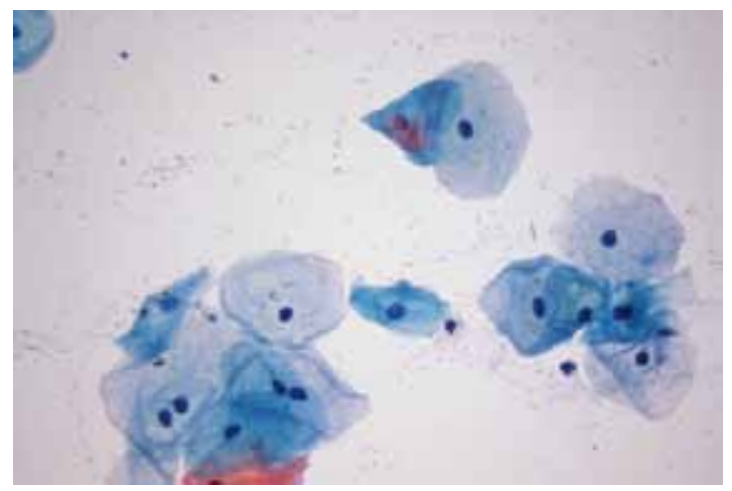

Figura 4. Células intermedias y superficiales en frotis vaginal de control con menor cantidad de lactobacilos que en frotis ilustrado en Figura 1. Papanicolaou, aumento 400x. en edad reproductiva, que generalmente consultan múltiples veces al ginecólogo, debido a un flujo vaginal persistente. No infrecuente que se les recete un antimicótico local, al confundirse con una micosis corriente por cándida albicans. Tampoco es infrecuente, que ante la nula respuesta frente al tratamiento, se cambien de médico, continuando con diferentes antimicóticos. Tal como sucedió en el caso descrito. Por lo tanto debemos sospecharla en quien nos relata que a pesar de múltiples tratamientos antimicóticos, continúa con los síntomas. La vaginosis citolítica se presenta con prurito, ardor y frecuentemente con dispareunia. Estos síntomas aumentan durante la fase lútea.

El exceso de lactobacilos se conoce como lactobacilosis, la cual puede llevar a bacteremia (15) e incluso a septicemia en pacientes inmunocomprometidos (16). Deficiencias o alteraciones del complemento o de inmunoglobulinas, podrían ser la causa del desarrollo de VC. La lactobacilosis vaginal se asocia generalmente a lactobacilos extremadamente largos, y conocidos como leptothrix. La causa de esta transformación morfológica es desconocida y se asocia a descarga de flujo abundante, 7 a 10 días después de la menstruación (17).

Algunos estudios han demostrado que los lactobacilos son más abundantes en aquellas mujeres con glicemia elevadas $(18,19)$, y esta condición podría deberse al aumento de glicógeno en las paredes vaginales.

Los criterios diagnósticos incluyen la ausencia de tricomonas, gardnerella y cándida, un aumento de los lactobacilos, presencia escasa de leucocitos, evidencia de citólisis, presencia de descarga excesiva de flujo y un $\mathrm{pH}$ vaginal entre 3,5 y 4,5 (14). La descarga de flujo vaginal, el prurito y muchas veces una sensación de quemadura, suelen ser cíclicas (19) (Tabla I).

La VC frecuentemente se confunde con candidiasis vaginal (20). Un estudio demostró que en estas pacientes, se demuestran sobre 200 colonias de lactobacilos, con un score de Nugent de cero (21).

La VC puede ser causa de vulvodinia (22), que si no se sospecha e identifica, retrasará el tratamiento, provocando dispareunia, que teóricamente podría llevar al desarrollo de un vaginismo.

Importante en la confirmación diagnóstica es la observación por microscopía, ya que nos permitirá demostrar la presencia de citólisisis (13). Característica es la observación de células epiteliales fragmentadas y/o núcleos libres debido a la lisis (20). La citólisis es producto de la presencia abundante de flúor, probablemente debido a la mayor 
cantidad de ácido láctico, como también a la mayor producción de peróxido de hidrógeno (21). Los lactobacilos adheridos a las células fragmentadas pueden confundirse con las llamadas "clue cells" de las vaginosis bacterianas (22).

\section{Tabla I \\ CRITERIOS PARA EL DIAGNÓSTICO DE VAGINOSIS CITOLÍTICA}

Síntomas y signos: Prurito y/o ardor vaginal. Aumento de la descarga de flujo vaginal. Frecuentemente aumentan en la fase lútea, por lo que se trata de síntomas y signos que se exacerban cíclicamente.

Examen ginecológico: Descarga abundante de flujo de aspecto de leucorrea e irritación de la mucosa vaginal.

Cultivo vaginal: Desarrollo de flora comensal (lactobacilos). Ausencia de otros microorganismos patógenos como cándida albicans, tricomona, clamidias, entre otros patógenos, como causantes de los signos y síntomas vaginales.

Estudio de gram: Compatible con identificación de lactobacilos abundantes. Ausencia o escasos leucocitos.

Medición del $\mathrm{pH}$ vaginal: Anormalmente ácido, entre 3,5 y 4,5 . Teniendo presente que el $\mathrm{pH}$ normal es entre 4,5 y 5,5 .

Estudio por microscopia: Demostración en biopsia o extendido citológico, de lisis en las células epiteliales. Destaca la fragmentación celular y la presencia de núcleos fuera de la célula. Observación de abundantes lactobacilos, sueltos y adheridos a las células epiteliales.

Características frecuentes de los lactobacilos: Se observan cambios morfológicos, destacando la presencia de lactobacilos alargados (leptothrix).

Por otro lado, actualmente se han identificado toxinas específicas, con actividad citolítica en diferentes bacterias vaginales. Un ejemplo es la gardnerella vaginalis. Se trata de la vaginolisina, un citolítico específico del epitelio vaginal humano $(23,24)$. Tal vez, estos lactobacilos asociados a VC, que se sabe pueden tener cambios morfológicos, también son capaces de producir toxinas específicas, que pueden provocar la lisis celular.

En un estudio con 101 mujeres entre 15 y 50 años, que consultaron por flujo vaginal y/o mal olor, vaginosis bacteriana se diagnosticó en el $34 \%$ y candidiasis en el $23 \%$, Clamidia tracomatis $15 \%$, Tricomona $9 \%$, Herpes $7 \%$ y Neisseria gonorrhoeae $1 \%$; entre estas mujeres destaca que en el $5 \%$ se diagnosticó VC (25). Un estudio de 210 mujeres con flujo vaginal y otros signos y síntomas que sugerían una vulvovaginitis micótica, demostró que en el $7,1 \%$ de los casos correspondía a una VC (20). Otro estudio de 2.947 frotis vaginales, evidenció que en el 1,8\% de los casos se cumplía con los criterios citológicos de una vaginosis citolítica (26). Una revisión efectuado en trabajadoras sexuales, detectó VC en el 1,9\% (27).

Entre las medidas más importantes en el tratamiento, debemos controlar el $\mathrm{pH}$ ácido, para lo cual podemos utilizar lavados con bicarbonato de sodio. $(13,14,22)$. Un seguimiento de 47 mujeres con VC, demostró que después de 2 ciclos de duchas vaginales con bicarbonato de sodio, 32 mujeres presentaron una mejoría en sus síntomas y signos (21). Otra medida importante es el tratamiento con antibióticos, que pueden controlar o disminuir la población aumentada de lactobacilos $(17,20)$.

Algunos estudios han demostrado que ciertas mutaciones genéticas, pueden otorgar resistencia frente a algunos antibióticos. Un estudio realizado este año, demostró que una mutación en 23S rRNA, se asocia a resistencia a la eritromicina (28). Esta mutación también ha sido identificada en otras bacterias, tanto de la vagina (29) como de otros órganos, otorgando la misma resistencia a macrólidos (30).

Diferentes estudios han demostrado que el uso de probióticos, conteniendo lactobacilos, pueden disminuir la probabilidad de presentar vaginosis bacteriana, candidiasis vulvovaginal e infecciones urinarias $(31,32)$. Sin embargo, el uso indiscriminado de estos productos podría ser la razón del aumento anormal de lactobacilos vaginales, que eventualmente llevan a una vaginosis citolítica. En la literatura se han descrito algunos casos de sepsis por lactobacilos, asociados a terapias con prebióticos $(33,34)$.

\section{CONCLUSIÓN}

En casos de vulvovaginitis a repetición o de múltiples consultas por sospecha de micosis vaginal, con escasa respuesta frente a antimicóticos, debemos sospechar la presencia de vaginosis citolítica. La lactobacilosis y la vaginosis citolítica, son diagnósticos que pueden confundirse con vulvovaginitis micótica, debido a la similitud de síntomas y signos.

\section{BIBLIOGRAFÍA}

1. Anderson MR, Klink K, Cohrssen A. Evaluation of vaginal complains. JAMA 2004;291(11):1368-79.

2. Spegel CA. Vaginitis/vaginosis. Clin Lab Med 1989;9(3):525-33. 
3. Eschenbach DA. Vaginal infection. Clin Obstet Gynecol Gynecol 1983;26(1):186-202.

4. Garg KB, Ganguli I, Das R, Talwar GP. Spectrum of Lactobacillus species present in healthy vagina of Indian women. Indian J Med Res 2009;129(6):652-7.

5. Barrons R, Tassone D. Use of Lactobacillus probiotics for bacterial genitourinary infections in women: a review. Clin Ther 2008;30(3):453-68.

6. Osset J, García E, Bartolomé RM, Andreu A. Role of Lactobacillus as protector against vaginal candidiasis. Med Clin (Barc) 2001;117(8):285-8.

7. Osset J, García E, Bartolomé RM, Andreu A. Assessment of the capacity of Lactobacillus to inhibit the growth of uropathogens and block adhesion to vaginal epithelial cells. J Infect Dis 2001;183(3):485-91.

8. Boris $\mathrm{S}$, Barbés $\mathrm{C}$. Role played by lactobacilli in controlling the population of vaginal pathogens. Microbes Infect 2000;2(5):543-6.

9. Lepargneur JP, Rosseau V. Protective role of the Doderlëin flora. J Gynecol Obstet Biol Reprod (Paris) 2002;31(5):485-94.

10. Dimitonova SP, Bakalov BV, Aleksandrova-Georgieva RN, Danova ST. Phenotypic and molecular identification of lactobacilli isolated from vaginal secretions. $J$ Microbiol Immunol Infect 2008;41(6):469-77.

11. Líbalová Z, Cepický P, Malina J, Stanslický K, Kuzelová Z, Medalová Z, et al. Vulvovaginitis. Occurrence and importance of mixed and unclassifiable pictures. Ceska Gynekol 2007;72(1):32-7.

12. Ramírez-Santos A, Pereiro Jr M, Toribio J. Vulvovaginitis de repetición. Valoración diagnóstica y manejo terapéutico. Actas Dermosifiliogr 2008;99:190-8.

13. Secor RM. Cytolytic vaginosis: a common cause of cyclic vulvovaginitis. Nurse Pract Forum 1992;3(3):145-8.

14. Cibley LJ, Cibley LJ. Cytolytic vaginosis. Am J Obstet Gynecol 1991;165(4 Pt 2):1245-9.

15. Husni RN, Gordon SM, Washington JA, Longworth DL. Lactobacillus bacteremia and endocarditis: review of 45 cases. Clin Infect Dis 1997;25(5):1048-55.

16. Shopova E. Lactobacillus spp. as part of the normal microflora and as pathogens in humans. Akush Ginekol (Sofia) 2001;42(2):22-5.

17. Horowitz BJ, Mårdh PA, Nagy E, Rank EL. Vaginal lactobacillosis. Am J Obstet Gynecol 1994;170(3):857-61.

18. Galask RP. Vaginal colonization by bacteria and yeast. Am J Obstet Gynecol 1998;158(4):993-5.

19. Hutti MH, Hoffman C. Cytolytic vaginosis: an overlooked cause of cyclic vaginal itching and burning. $J$ Am Acad Nurse Pract 2000;12(2):55-7.

20. Cerikcioglu N, Beksac MS. Cytolytic vaginosis: misdiagnosed as candidal vaginitis. Infect Dis Obstet Gynecol 2004;12(1):13-6.
21. Shopova E, Tiufekchieva E, Karag'ozov I, Koleva V. Cytolytic vaginosis-- clinical and microbiological study. Akush Ginekol (Sofiia) 2006;45 Suppl 2:12-3.

22. Paavonen J. Vulvodynia --a complex síndrome of vulvar pain. Acta Obstet Gynecol Scand 1995;74(4):243-7.

23. Gelber SE, Aguilar JL, Lewis KL, Ratner AJ. Functional and phylogenetic characterization of Vaginolysin, the human-specific cytolysin from Gardnerella vaginalis. J Bacteriol 2008;190(11):3896-903.

24. Randis TM, Kulkarni R, Aguilar JL, Ratner AJ. Antobody-based detection and inhibition of vaginolysin, the Gardnerella vaginalis cytolysin. PLoS One 2009;4(4):e5207.

25. Wathne B, Holst E, Hovelius B, Mårdh PA. Vaginal discharge-comparison of clinical, laboratory and microbiological findings. Acta Obstet Gynecol Scand 1994;73(10):802-8.

26. Demirezen S. Cytolytic vaginosis: examination of 2947 vaginal smears. Cent Eur J Public Health 2003;11(1):23-4.

27. Amaral R, Giraldo PC, Gonçalves AK, Junior JE, Santos-Pereira S, Linhares I, et al. Evaluation of hygienic douching on the vaginal microflora of female sex workers. Int J STD AIDS 2007;18(11):770-3.

28. Begovic J, Huys G, Mayo B, D'Haene K, Florez $A B$, Lozo $\mathrm{J}$, et al. Human vaginal Lactobacillus rhamnosus harbor mutation in 23S rRNA associated with erythromycin resistance. Res Microbiol 2009;160(6):421-6.

29. Pereyre S, Gonzalez P, De Barbeyrac B, Carnige A, Renaudin $\mathrm{H}$, Charron $\mathrm{A}$, et al. Mutation in 23S rRNA account for intrinsic resistance to macrolides in Mycoplasma hominis and Mycoplasma fermentans and for acquired resistance to macrolides in M. hominis. Antimicrob Agents Chemother 2002;46(10):3142-50.

30. Lee SY, Ning Y, Fenno JC. 23 S rRNA point mutation associated with erythromycin resistance in Treponema denticola. FEMS Microbiol Lett 2002;207(1):39-42.

31. Abad CL, Safdar N. The rol of lactobacillus probiotics in the treatment or prevention of urogenital infections--a systematic review. J Chemother 2009;21(3):243-52.

32. Falagas ME, Betsi GI, Athanasiou S. Probiotics for prevention of recurrent vulvovaginal candidiasis: a review. J Antimicrob Chemother 2006;58(2):266-72.

33. Land MH, Rouster-Stevens K, Woods CR, Cannon ML, Cnota J, Shetty AK. Lactobacillus sepsis associated with probiotic therapy. Pediatrics 2005;115(1):178-81.

34. Zein EF, Karaa S, Chemaly A, Saidi I, Daou-Chahine W, Rohban R.Lactobacillus rhamnosus septicemia in a diabetic associated with probiotic use: a case report. Ann Biol Clin (Paris) 2008;66(2):195-8. 